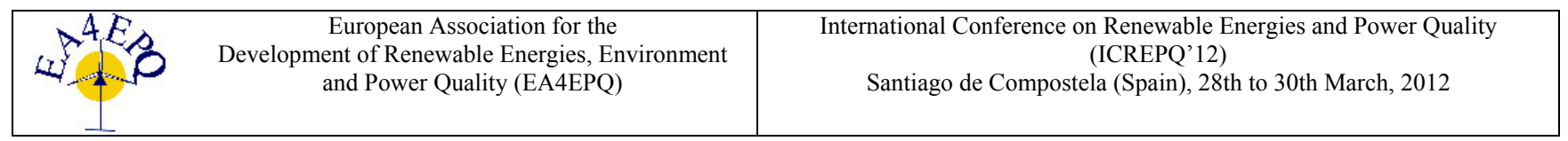

\title{
Vertical Axis Wind Turbine Performance Prediction: An Approach to the Double Multiple Streamtube Model
}

\author{
N.C. Batista ${ }^{1}$, R. Melício ${ }^{1,2}$, J.C.O. Matias ${ }^{1}$ and J.P.S. Catalão ${ }^{1,2}$ \\ ${ }^{1}$ University of Beira Interior and CAST \\ R. Fonte do Lameiro, 6200-001 Covilhã (Portugal) \\ Phone: +351275329 914, Fax: +351275329972 \\ e-mail: nelson.batista@gmail.com, ruimelicio@gmail.com, matias@ubi.pt \\ ${ }^{2}$ Center for Innovation in Electrical and Energy Engineering, Instituto Superior Técnico \\ Av. Rovisco Pais 1, 1049-001 Lisbon (Portugal) \\ Phone: +351218 417 000, Fax: +351218 499242 \\ e-mail: catalao@ubi.pt
}

\begin{abstract}
The vertical axis wind turbines (VAWT) have been suffering an increased acceptance for urban wind turbines integration in the future smart grid for decentralized generation (DG). The VAWT have several advantages over the more conventional horizontal axis wind turbines (HAWT): the smaller number of components; low sound emissions; their insensitivity to fast changes in wind flow direction; a lower architectural visual impact; the ability to operate closer to the ground; and others. But, the VAWT aerodynamic performance is more complex to simulate and predict due to its three dimensional operation. Several mathematical models have been presented to predict the VAWT performance, such as the double multiple streamtube (DMS) model. With the aim of presenting a tool to ease the study of complex shaped VAWTs, being at the same time able to be easily integrated in computational design tools, a new approach to the DMS model is presented in this paper.
\end{abstract}

\section{Key words}

Vertical axis wind turbine, performance prediction, double multiple stremtube model.

\section{Introduction}

The use of fuel sources with high $\mathrm{CO}_{2}$ emissions is raising and the need for cleaner resources in a renewed grid is increasing, being the wind energy one of the best choices [1]. In the future smart grid, the integration of DG in urban areas is needed. The wind is one of the best energy sources available in urban areas [2], having the VAWT several advantages over the HAWT [3].

Several VAWT solutions exist in the market, but they lack of technological innovation, presenting different configurations of already existing solutions. This is due to the high complexity of the VAWT aerodynamic prediction, leading to a lack of industrial I\&D new developments.
This paper offers several prediction tools and brings an approach to the VAWT performance evaluation in complex shaped VAWT, being at the same time easily integrated as an extra module in Computer-Aided Design (CAD) and Computational Fluid Dynamics (CFD) existing software solutions.

\section{Approach to DMS}

The new approach proposed is based on the DMS VAWT performance prediction model from Paraschivoiu [4]-[5], in which the wind turbine is divided in several parallel and adjacent streamtubes that are considered to be independent from each other, having their own wake and induced velocities, as shown in Figure 1.

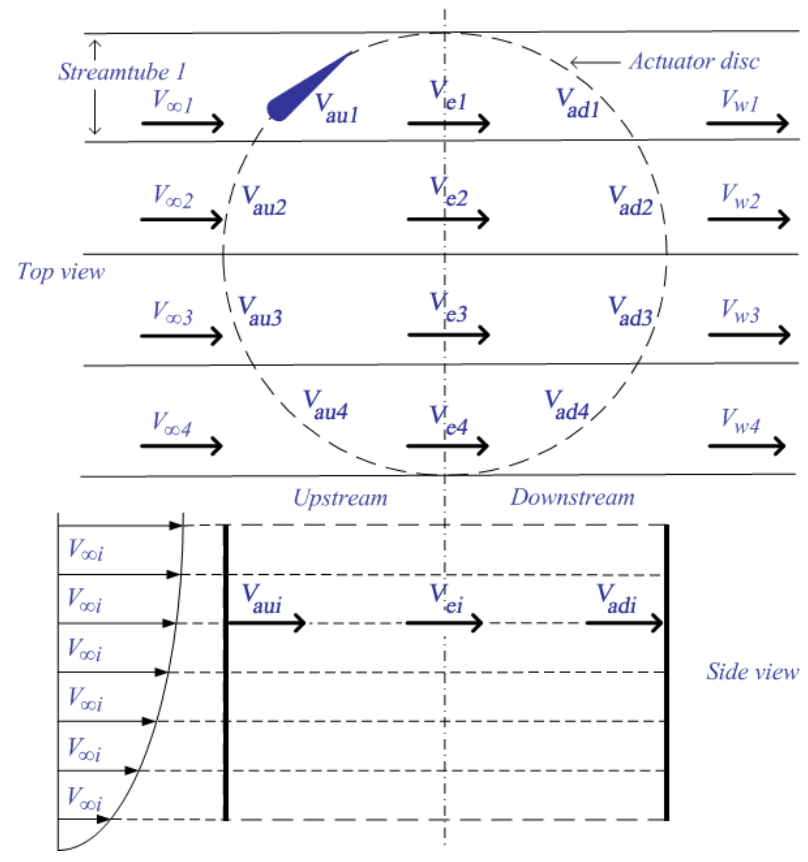

Fig. 1. DMS model diagram. 
The rotor movement is translated in an actuator disc divided in two half cycles, representing the downstream and upstream sides of the rotor.

For this paper no modifications are presented to the DMS model, but instead a new approach to the use of this model to study the performance prediction of complex VAWT blades configurations is presented.

The difference of our new approach to the normal use of the DMS model is that, instead of directly integrating all the streamtubes data at the same time, first the wind turbine rotor space is divided in several slices parallel to the wind flow direction, and then each slice is threatened as an independent wind VAWT and evaluated.

\section{A. The DMS Model}

Despite the complexity of the aerodynamic behaviour of the Darrieus VAWT, several mathematical models have been presented, and all have their strengths and weaknesses and have a more or less accurate prediction, depending on the wind turbine configuration, wind behaviour and prediction time consumed.

The most used models can be divided in three categories: Vortex model, Cascade model and Blade Element Momentum model.

The Blade Element Momentum (BEM) model combines momentum theory with blade element theory. It studies the behaviour of the air flow on the blades and its forces.

The BEM model can be further divided in the models: Single Streamtube model, Multiple Streamtube model and Double-Multiple Streamtube model.

In the Single Streamtube model the turbine is placed inside a single streamtube and its blades revolution are translated in an actuator disk. The effects of the streamtube outside are assumed negligible and the wind speed in the upstream and downstream sides of the turbine are assumed to be constant. This model does not have a good turbine performance prediction, which is the result of all the assumptions made; it usually gives higher prediction values.

The Multiple Streamtube model is a variation of the Single Streamtube model, where the single streamtube is divided in several parallel adjacent streamtubes that are independent from each other and have their own undisrupted, wake and induced velocities.

Several modifications have been presented for this model where the drag forces, aerofoil geometry, curvature flow, etc. where added, but still its predictions are away from experimental data.

The Double Multiple Streamtube (DMS) model is a variation of the Multiple Streamtube model, where the actuator disc is divided in two half cycles in tandem, representing the upstream and downstream sides of the rotor. This model was presented by Paraschivoiu [4]-[5].
Figure 1 presented the DMS model diagram. The actuator disc is divided in two actuator disc, each of them with their own induced velocity.

The induced velocity decreases along the axial streamtube direction, so the induced velocity in the upstream $V_{a u i}$ is less than the undisrupted wind speed $V_{\infty i}$ that arrives to the streamtube $i$.

Between the upstream and the downstream there is an equilibrium induced velocity $V_{e i}$ that is less than the $V_{a u i}$.

The induced velocity in the downstream $V_{a d}$ is less than $V_{e i}$. So for the induced velocity in the upstream $V_{a u i}$ we get:

$V_{a u}=V_{\infty i} u_{u s}$

where $u_{u s}$ is the interference factor for the upstream which is less than 1 and is given by:

$u_{u s}=\frac{V_{a u}}{V_{\infty i}}$

The induced velocity in the downstream $V_{e}$ is influenced by the wake velocity of the upstream, which is given by:

$V_{e}=V_{\infty i}\left(2 \frac{V_{a u}}{V_{\infty i}}-1\right)=V_{\infty i}\left(2 u_{u s}-1\right)$

The induced velocity in the downstream $V_{a d}$ is given by:

$V_{a d}=u_{d s} V_{e}=u_{d s}\left(2 u_{u s}-1\right) V_{\infty i}$

where $u_{d s}$ is the interference factor for the downstream. The $u_{d s}$ is given by:

$u_{d s}=\frac{V_{a d}}{V_{e}}$

As it can be seen the aerodynamic behaviour of the blades in the upstream side of the wind turbine will influence the induced velocity on the blades in the downstream side.

The undisrupted wind velocity $V_{\infty i}$ is defined by the wind velocity profile and increases along the wind turbine height.

By applying the DMS prediction model with the VAWT performance equations presented previously, is possible to predict the turbine performance.

The torque and power coefficient are found by integrating the aerodynamic behaviours of the several streamtubes. 
For the wind turbines that differ from the straight bladed VAWTs, the local blades radius and incline are adjusted in relation to the blade form in the streamtube that is under evaluation and will influence the airfoil aerodynamic properties. This is the case of the performance evaluation of the egg shaped VAWT.

\section{B. New Approach}

The VAWT is divided in several slices that are parallel to the wind flow path, as shown in Figure 2 and Figure 3.

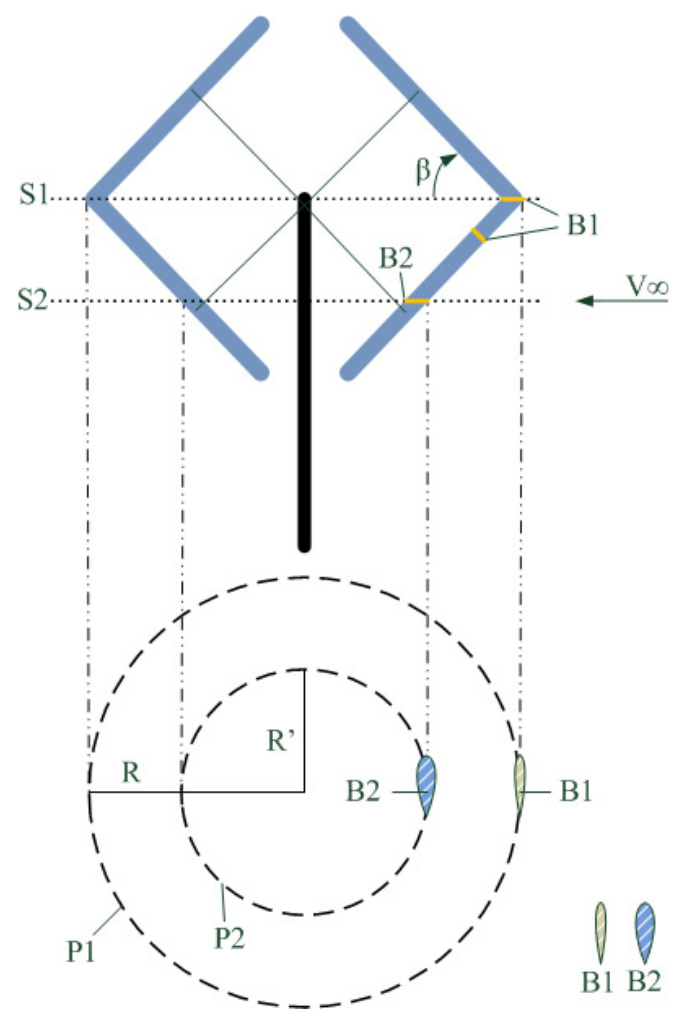

Fig. 2. DMS model approach applied to a V shaped Darrieus wind turbine.

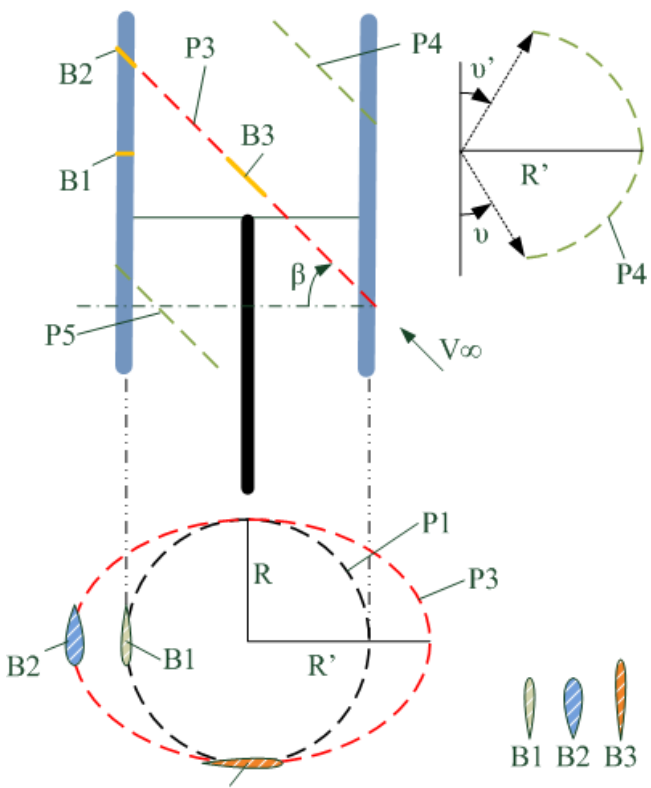

B3

Fig. 3. DMS model approach applied to an H-Rotor Darrieus wind turbine in skewed flow.
In each slice the blade profile shape design is analysed for each axial angle in the blade movement path.

As a visual help we have the blade movement paths resulting from slice S1 and slice S2 (Figure 2) that are represented as $\mathrm{P} 1$ and $\mathrm{P} 2$ correspondently.

The Lift and Drag are calculated for each of the blade profile designs on each of the axial angles.

It must be point out that depending on the slice positioning, the wind can be presented with different bade designs for the same VAWT blade.

As shown in Figure 2, the same VAWT blade has two different profile designs, one when analysing slice S1 and other for slice $\mathrm{S} 2$.

Although the VAWT blade is the same, it presents two different blade profiles $\mathrm{B} 1$ and $\mathrm{B} 2$ for slice $\mathrm{S} 1$ and $\mathrm{S} 2$ respectively.

Even in the same slice as shown in Figure 3, the blade can present different aerofoil designs as with path P3. In this path it can be seen that the blade profiles B2 and B3 are different from the VAWT blade profile B1 that would be used in the normal DMS model for the performance prediction.

The blade profile $\mathrm{B} 2$ has a higher body than the blade profile $\mathrm{B} 1$ and the aerofoil B3 is wider.

One remark must be made, not only the blade design is analysed but also the radius $\mathrm{R}$ and $\mathrm{R}$ ' are analysed for each axial angle, leading to different performance output data, as for example the analysis of the VAWT performance made under skewed flows (Figure 3).

In each slice, the blade existence in a certain axial angle must be taken in consideration, as shown with path P4, where not all the angles are considered.

All of these analyses of the VAWT blade existence in each axial angle for each slice go largely away from the normal DMS model use, but it leads to a more accurate performance prediction and offers the ability to use this mathematical model with more complex shaped VAWTs.

Although it seems very time consuming to create all the slices parallel to the air flow path, and analysing the blade positioning, form, radius and behaviour, with the help of CAD and CFD this task is today simplified with the computational advances and has a reasonable performance.

This new approach offers the ability to easily apply this performance model to any kind of VAWT with or without complex shaped blades and at any flow conditions, which represents a great advance in the applicability of the DMS model. Further advantages with this approach to the DMS model, is the ability to implement it on CAD and CFD software simplifying the work flow of the engineers. 


\section{Conclusion}

This paper focus on the study and development of new Darrieus type VAWT, by applying a new approach to the DMS prediction model. Along with the presentation of the VAWT DMS model, a new approach to it is introduced by using wind turbine slices parallel to the wind flow. In each slice the form and behaviour of the blade as it is found by the wind flow is studied. This new approach offers the ability to easily apply this performance model to any kind of VAWT with or without complex shaped blades and at any flow conditions, which represents a great advance in the applicability of the DMS model. Further advantages with this approach to the DMS model, is the ability to implement it on CAD and CFD software simplifying the work flow of the engineers. Hence, this paper offers fast and solid tools for new VAWT developments.

\section{References}

[1] A. F. Zobaa and B. K. Bose, "Renewable energy, global warming problem and impact of power electronics", in: ICREPQ'11, Gran Canaria, Spain, April 2011.

[2] M. Mañana, "Small wind energy systems. State of the art and new challenges", in: ICREPQ'11, Gran Canaria, Spain, April 2011.

[3] N.C. Batista, R. Melício, J.C.O. Matias, J.P.S. Catalão, "Self-start performance evaluation in Darrieus-type vertical axis wind turbines: Methodology and computational tool applied to symmetrical airfoils", in: ICREPQ'11, Gran Canaria, Spain, April 2011.

[4] I. Paraschivoiu, "Double-multiple streamtube model for studying vertical-axis wind turbines", Journal of Propulsion and Power, Vol. 4, No. 4, pp. 370-377, JulAug 1988.

[5] I. Paraschivoiu, "Wind turbine design: with emphasis on Darrieus concept", Polytechnic International Press, Canada; 1st edition, June 2002. 